\title{
Diagnosis of Articular Disc Perforation: A Case Series
}

\author{
Daeun $\mathrm{Ko}^{1}$, Hyun Nam ${ }^{1}$, Young-Joo Shim ${ }^{1,2}$, Jin-Kyu Kang ${ }^{1,2}$ \\ ${ }^{1}$ Department of Orofacial Pain and Oral Medicine, Wonkwang University Daejeon Dental Hospital, Daejeon, Korea \\ ${ }^{2}$ Wonkwang Dental Research Institute, Wonkwang University, Iksan, Korea
}

Received December 3, 2019

Revised December 16, 2019

Accepted December 16, 2019

\section{Correspondence to:}

Jin-Kyu Kang

Department of Orofacial Pain and Oral

Medicine, Wonkwang University Daejeon

Dental Hospital, 77 Dunsan-ro, Seo-gu,

Daejeon 35233, Korea

Tel: +82-42-366-1125

Fax: +82-42-366-1115

E-mail: orofacial@wku.ac.kr

https://orcid.org/0000-0001-7668-7468

This paper was supported by Wonkwang University in 2019.
Perforation of articular disc of temporomandibular joint is a unusual condition and diagnosed through magnetic resonance imaging (MRI), arthrography or arthroscopic surgery. We attempted to investigate the suspicious findings of articular disc perforation through examination commonly used in temporomandibular disorder (TMD) patients. We retrospectively analyzed the clinical and imaging findings of five TMD patients whose articular discs were perforated based on MRI. The most meaningful finding was the abnormal width of the joint space in cone-beam computed tomography. Thus, the clinician should perform a thorough assessment of the joint space in TMD patients and conduct additional investigation to determine what caused the abnormal joint space.

Key Words: Cone-beam computed tomography; Magnetic resonance imaging; Temporomandibular joint disc

\section{INTRODUCTION}

Articular disc perforationof temporomandibular joint (TMJ) is a unusual form of the intra-articular disorder, which usually occurs with existing disc disorder (i.e. disc displacement) or late stage of osteoarthritis [1]. It is reported to be seen in $8.2 \%$ of disc displacement patients [2], and the incidence rate increases with age [3]. Disc perforation causes direct contact of articular surfaces between the condyle and fossa. It alters the synovial fluid secretion, increases intra-articular friction, and provokes the degeneration of intra-articular structure [3]. Therefore, disc perforation presumably acts as an adverse prognostic factor for previously existing temporomandibular disorders (TMDs).

Since there is no specific signs and symptoms of disc perforation, clinical diagnosis of disc perforation of TMJ is challenging $[1,4,5]$. However, thorough clinical examination and magnetic resonance imaging (MRI) may result in the diagnosis of disc perforation. Here, we report five cases of disc perforation and review the clinical and imaging findings with review of literatures.

\section{CASE REPORT}

\section{Case 1}

An 83-year-old female visited with pain on her left jaw during mastication lasting about six months.

Clinical examination showed the active mouth opening of $33 \mathrm{~mm}$ with pain, crepitus, and tenderness to palpation on the left TMJ. The occlusal contacts were stable on both sides. Cone-beam computed tomography (CBCT) showed generalized sclerosis, osteophyte formation and narrowed anterosuperior joint space on the left TMJ (Fig. 1A). Disc deformity was suspected by severely narrowed joint space and MRI was taken to evaluate the integrity of the intraarticular structure.

The T2-weighted MRI revealed the loss of continuity of the posterior attachment, effusion, and medially displaced 

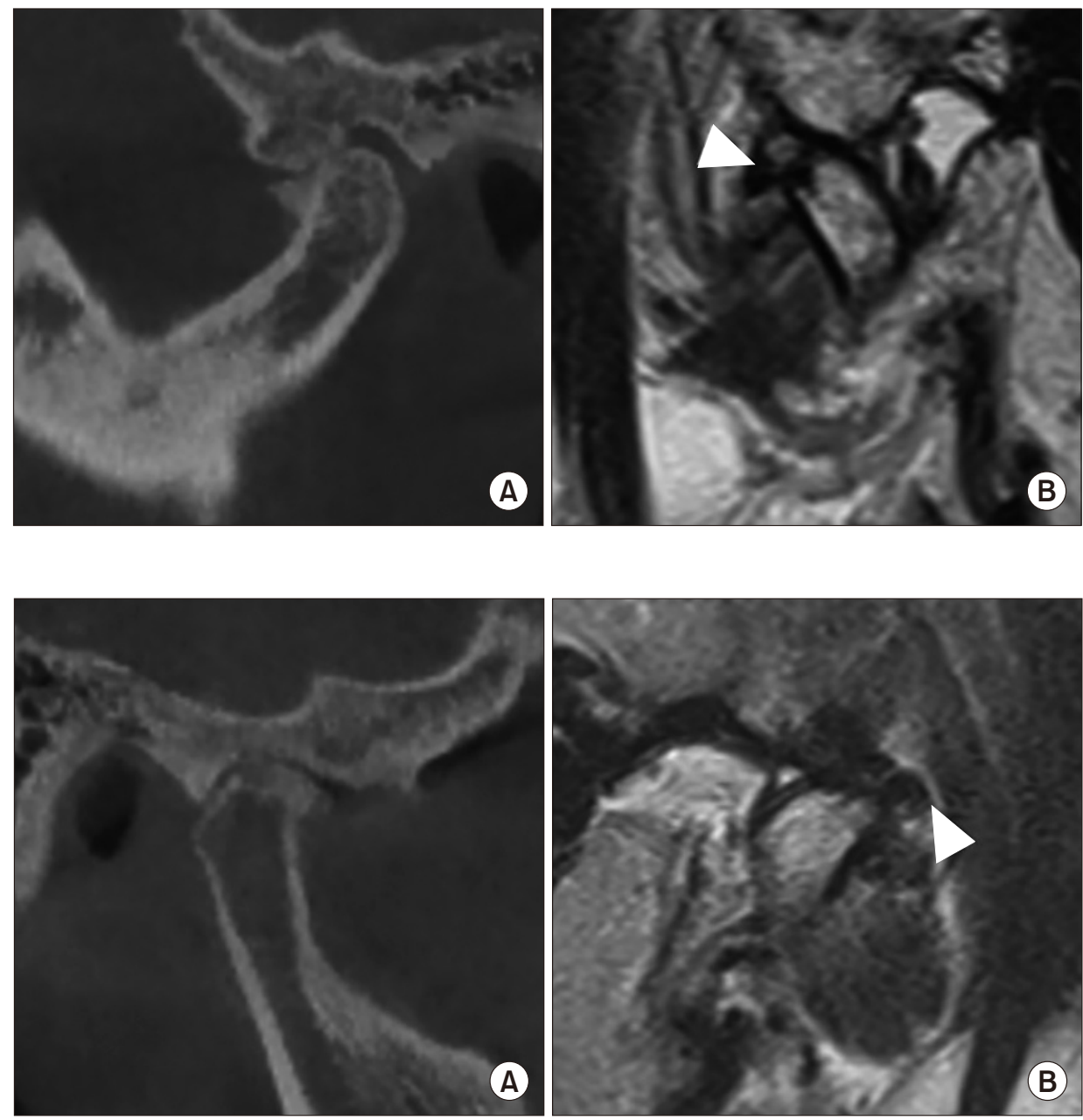

Fig. 1. Cone-beam computed tomography $(\mathrm{CBCT})$ and $\mathrm{T} 2$-weighted magnetic resonance (MR) images of Case 1. (A) The sagittal CBCT shows generalized sclerosis, osteophyte formation, and narrowed anterosuperior joint space on the left temporomandibular joint. (B) The sagittal MR image shows loss of continuity of the posterior attachment of the disc, effusion, and medially displaced disc, which was perforated in the anteroposterior direction in the maximum opening position. The arrowhead indicates the perforated disc.

Fig. 2. Cone-beam computed tomography (CBCT) and T2-weighted magnetic resonance (MR) images of Case 2. (A) The sagittal CBCT shows sclerosis, subchondral bone cyst, osteophyte formation, and uniformly narrowed joint space on the right temporomandibular joint. (B) The sagittal MR image shows loss of continuity of the posterior attachmentand the disc. The posterior attachment is behind the condyle and the disc in front of the condylar head in the maximum opening position. The arrowhead indicates the perforated disc.

disc of the left joint at maximum mouth opening position. The disc was torn completely into two segments (Fig. 1B). No gross abnormality was shown in the right TMJ. Physiotherapy, pharmacotherapy, stabilization splint therapy, and arthrocentesis were performed. The pain was significantly relieved when compared to the initial visit for a 7-month follow-up period.

\section{Case 2}

A 57-year-old female presented with pain on her right preauricular area during jaw function and thesensation of being unable to bite as usual on the right molar teeth. Clinical examination showed the active mouth opening of $44 \mathrm{~mm}$ with pain, crepitus, and tenderness to palpation on the right TMJ. The occlusal contacts were stable except for her right second molar. CBCT presented sclerosis, subchondral bone cyst, osteophyte formation, and uniformly narrowed joint space on the right TMJ (Fig. 2A).
In the T2-weighted MRI, the continuity between the disc and the posterior attachment was lost on the right side at the maximum mouth opening position (Fig. 2B). Physiotherapy, pharmacotherapy, and stabilization splint therapy were offered and effective in reducing her symptom for a 6-month follow-up period. As the pain relieved, her occlusal contact was stabilized.

\section{Case 3}

A 62-year-old female complained pain on her right jaw during mouth opening and mastication. On the clinical examination, the active mouth opening was $45 \mathrm{~mm}$ with pain and crepitus on the right side. Non-specific finding was identified on her occlusal contacts. CBCT showed erosion and loose body on the right TMJ (Fig. 3A). And the vertical dimension of condyle and eminence was reduced, which resultedinwidened joint space in the right TMJ. MRI was taken to differentiate primary synovial chondromatosis from 

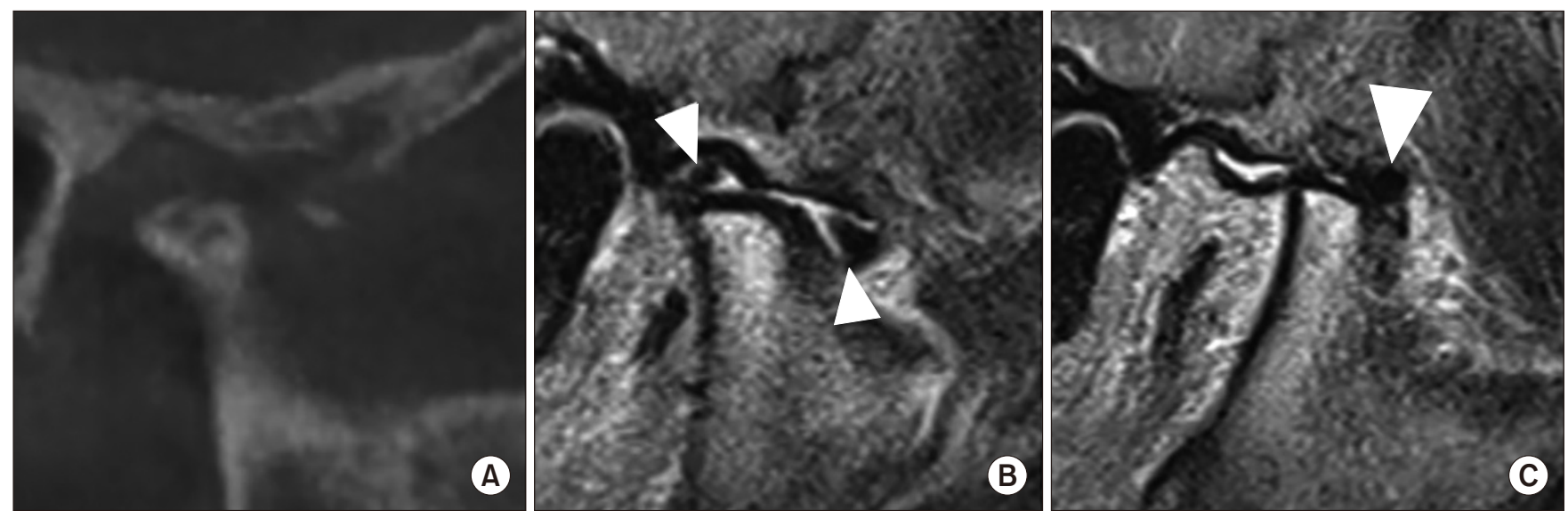

Fig. 3. Cone-beam computed tomography (CBCT) and T2-weighted magnetic resonance (MR) images of Case 3. (A) The sagittal CBCT shows erosion, reduced vertical dimension of articular eminence and condyle, and the loose body on the right temporomandibular joint. (B) The sagittal MR image shows disc perforation in an anteroposterior direction completely along with effusionin the closed-mouth position. (C) The sagittal MR image in the maximum opening position shows the articular eminence and the condyle are nearly close. The arrowheads indicate the perforated disc.
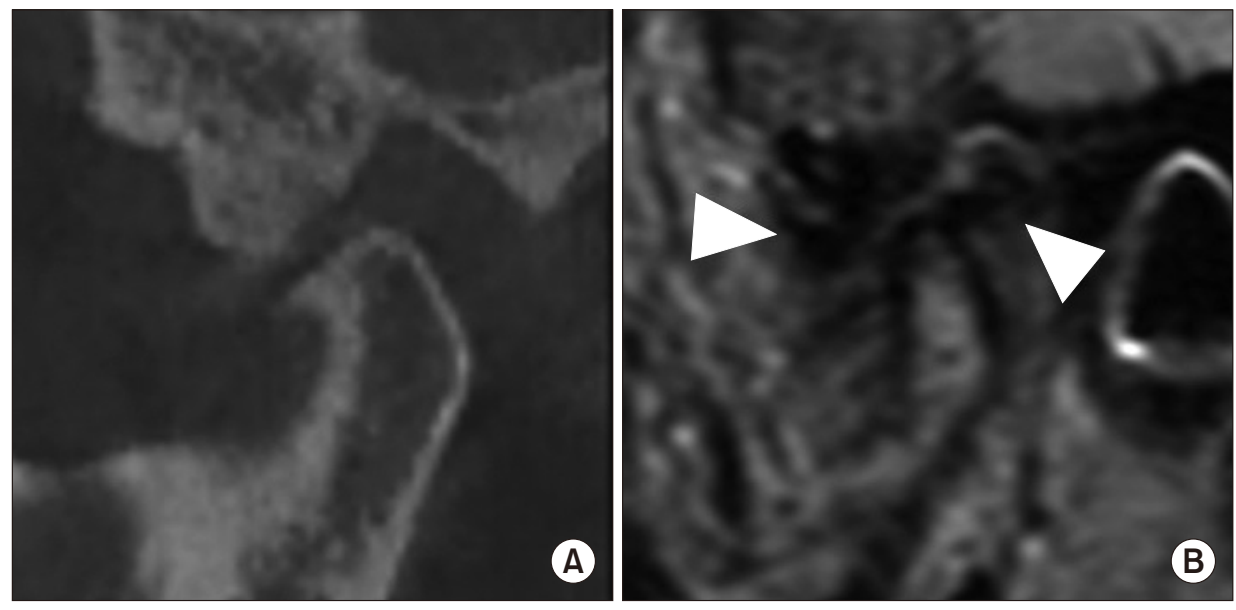

Fig. 4. Cone-beam computed tomography $(C B C T)$ and $T 2$-weighted magnetic resonance (MR) images of Case 4. (A) The sagittal CBCT shows widened joint space between the left condylar head and glenoid fossa with the slight degenerative changes. (B) The sagittal MR image shows the torn disc was found on the lateral aspect of the condylar head in the closed-mouth position. The arrowheads indicate the perforated disc.

degenerative joint disorder, due to the presence of the loose body.

In T2-weighted MRI, the disc was perforated completely in an anteroposterior direction along with effusion in right TMJ at the closed-mouth position (Fig. 3B). Patient education, physiotherapy, pharmacotherapy, and stabilization splint therapy have alleviated her symptoms for about 5-year follow-up period.

\section{Case 4}

A 69-year-old male complained of being unable to bite as usual on the left side. He reported it had lasted for about 1.5 months and accompanied by pain on the left jaw during functioning. Clinical examination showed the active mouth opening was $45 \mathrm{~mm}$ with pain, crepitus, and the tenderness to palpation on the left TMJ. The occlusal contacts of all the left teeth were lost, and those of the right side were stable. CBCT showed widening of joint space between the left condylar head and glenoid fossa with the slight degenerative changes (Fig. 4A).

In T2-weighted MRI, on the left TMJ, the torn disc was found on the lateral aspect of the condylar head, contrary to the medial aspect, where the integrity of the disc was conserved (Fig. 4B). The posterior segment from the perforated disc disrupted the condyle from going back to its normal position. Physiotherapy, pharmacotherapy, and manipulation were offered for about 6-month follow-up period. His jaw pain was significantly reduced compared to the initial visit. But the posterior open bite of the affected side has persisted refractory to those treatments. 


\section{Case 5}

A 76-year-old female patient complained she couldn't bite on her left teeth and had pain on the left jaw during functioning. She reported it had developed after chewing hard food and had lasted for about 1 month. The active mouth opening was $45 \mathrm{~mm}$ with pain and clicking on the left side was found on clinical examination. On the left side, the only contact was detected at the incisor, contrary to the right side, where it as stable occlusal contacts. We discovered erosion and bony spur on the left condyle with widened joint space and the inferiorly positioned condylar head in the CBCT (Fig. 5A).

Further evaluation using MRI was done to find out causal factors inducing posterior open bite and rule out the presence of space-occupying mass. In T2-weighted MRI, we verified the partially torn disc with effusion shown in the lateral aspect of the left TMJ and the posterior fragment of the perforated disc displaced posteriorly, which disrupted the left condyle from returning to its original position at maximal intercuspation position (Fig. 5B). Patient education, physiotherapy, and pharmacotherapy were offered for 1-month follow-up period. Those were ineffective to the occlusal change otherwise than her jaw pain. Additional treatment options, including prosthetic, orthodontic, and surgical treatment, were discussed. Owing to uncertainty on the outcome of those options, the patient declined to try it.

\section{DISCUSSION}

We retrospectively reviewed five TMD patients (4 females and 1 male) whose articular discs were confirmed perforated in MRI. Clinical features of five patients are summarized in Table 1. Three of them presented with jaw pain primarily during mastication and mouth opening, and the others were presented with being unable to bite on posterior teeth as usual. According to the previous studies $[3,6]$, the most
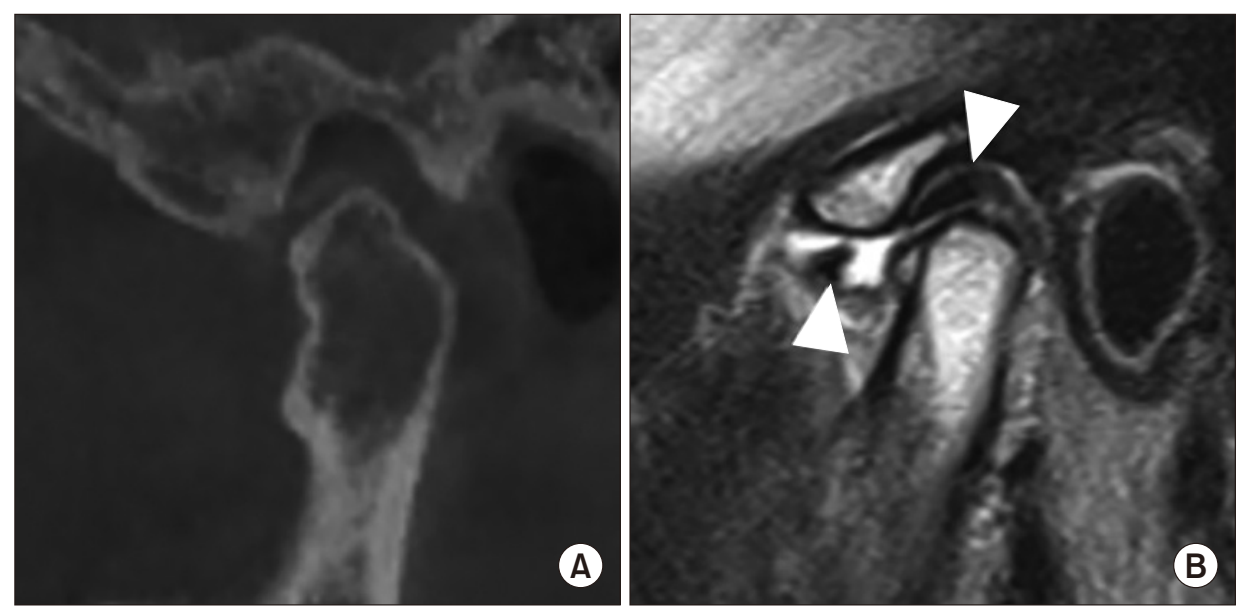

Fig. 5. Cone-beam computed tomography $(C B C T)$ and $T 2$-weighted magnetic resonance (MR) images of Case 5. (A) The sagittal CBCT shows joint space widened with the slight degenerative changes. (B) The sagittal MR image shows the torn disc with effusion and the posterior fragment of the perforated disc displaced posteriorly in the closed-mouth position. The arrowheads indicate the perforated disc.

Table 1. Clinical features of five patients

\begin{tabular}{|c|c|c|c|c|c|}
\hline & Case 1 & Case 2 & Case 3 & Case 4 & Case 5 \\
\hline Age/sex & $83 /$ female & 57/female & $62 /$ female & $69 /$ male & 76/female \\
\hline Follow-up period & $7 \mathrm{mo}$ & $6 \mathrm{mo}$ & $5 y$ & $6 \mathrm{mo}$ & $1 \mathrm{mo}$ \\
\hline Primary symptom & \multicolumn{3}{|c|}{ Joint pain } & \multicolumn{2}{|c|}{ Ipsilateral posterior open bite } \\
\hline $\begin{array}{l}\text { Radiographic } \\
\text { joint space }\end{array}$ & \multicolumn{3}{|c|}{ Narrowed joint space group } & \multicolumn{2}{|c|}{ Widened joint space group } \\
\hline Treatment & \multicolumn{3}{|c|}{$\begin{array}{l}\text { Physiotherapy, pharmacotherapy, stabilization splint, } \\
\text { arthrocentesis (in Case 1) }\end{array}$} & \multicolumn{2}{|c|}{$\begin{array}{l}\text { Physiotherapy, pharmacotherapy, } \\
\text { manipulation (in Case 4) }\end{array}$} \\
\hline Outcome & \multicolumn{3}{|c|}{ Reduced pain and dysfunction } & \multicolumn{2}{|c|}{ No effect on the occlusal change } \\
\hline Treatment plan & \multicolumn{2}{|c|}{ Periodic follow-up } & & \multicolumn{2}{|c|}{$\begin{array}{l}\text { Temporomandibular joint surgery } \\
\text { such as discectomy } \\
\text { Occlusal adjustment } \\
\text { Prosthetic or orthodontic treatment }\end{array}$} \\
\hline
\end{tabular}


common symptom in patients with articular disc perforation was joint pain and followed by the joint sound (crepitation and/or clicking) and limitation of mouth opening. These symptoms are not significantly different from those of other more prevalent TMDs. Thus, in general, there is no specific sign or symptom toward disc perforation. There are several articles [5,7], including our fifth case of this paper, reporting occlusal changes caused by articular disc perforation, although this is not generally expected clinical manifestation from articular disc perforation. It is also considered a subtype of posterior disc displacement, which refers to the posterior segment of the perforated disc displace posteriorly [4].

In $\mathrm{CBCT}$, a perforated disc is indirectly revealed by the finding the uniformly narrowed radiographic joint space, which results from loss of intercalating between fossa and condylar head [8]. Narrowed joint space was reported as one of the significant risk factors in predicting the possibility of disc perforation by Kim et al. [9]. The narrower the joint space becomes, the more likely the joint has the perforated disc. And the probability is higher when there is no space between condyle and eminence in the mouth opening position, referred to as bone-to-bone contact.

The findings of CBCT in five patients, which were taken in the closed-mouth position were reviewed. All five patients have degenerative bone changes in the condyle. The five patients divided into two groups according to the radiographic joint space width. Case 1, 2, and 3 were into the narrowed joint space group and Case 4 and 5 were into the widened joint space group. In Case 1 and 2, the articular eminence and condyle are very close to each other in $\mathrm{CBCT}$, and the distance is almost faint, and making it boneto-bone contact in maximum opening position of MRI. In Case 3, radiographic joint space is widened in the CBCT, which results from reduced vertical dimension of articular eminence and condyle. However, when observed in maximum opening position, the articular eminence and the condyle are nearly close in MRI (Fig. 3C). Therefore, Case 3 was classified into the narrowed joint space group. Although joint space narrowing is a common feature in degenerative joint disorder, the articular disc perforation frequently accompanies progressed degenerative arthritis. The presence of degenerative arthritis cannot exclude the possibility of perforation of the disc. In Case 4 and 5, the widened radiographic joint spaces were resultant from the posterior displacement of the posterior segment of the perforated disc. MRI evaluation is needed to identify the cause of the expanded joint space as well as to rule out the presence of space-occupying mass.

We mentioned that abnormal radiographic joint space could indirectly indicate the disc perforation. However, the exact normal range of joint space does not reported. One study [10] classified the width of joint space as a 'normal' range between $1.5 \mathrm{~mm}$ to $4 \mathrm{~mm}$, 'narrowed' if less than 1.5 $\mathrm{mm}$, and 'widened' if widened than $4 \mathrm{~mm}$. But, there was no relevant follow-up study and it is difficult to apply this classification. Furthermore, anatomical variations between patients make it more challenging to establish a consistent standard with a normal range of joint space. Generally, clinician evaluates the joint space by comparing it to the opposite side. In the case of abnormal joint space, MRI should be ordered, and the imaging and clinical findings should be evaluated comprehensively.

Definitive treatment of perforated disc is surgical intervention, including suture of perforated disc, discectomy, and disc replacement. However, the debatable outcome from surgery and unexpected side effects have led to trying conservative treatment before surgical treatment. Minimally invasive surgical options, such as arthrocentesis and arthroscopic surgery, are reported to be effective in reducing pain and dysfunction [11]. Thus, invasive open TMJ surgery should only be considered when other treatment fails. Occlusal treatment such as occlusal adjustment, orthodontic or prosthetic treatment may be considered when patients report uncomfortable occlusion. In this case series, the pain was well-controlled by conservative treatment. However, posterior open bite, as a complication by intra-articular structural incompatibility with disc perforation, was not resolved in spite of the repetitive conservative treatments. Clinicians dealing with this issue should discuss additional treatment options with patients and orthodontists or prosthodontists.

In conclusion, disc perforation is a rare condition and difficult to diagnose in commonly used protocols, rather it is usually found by chance. We aimed to discover features that may be suspected of disc perforation and one such 
finding is the width of the joint space. When a TMD patient has an abnormal joint space, it is recommended to order MRI to differentiate the underlying cause to establish a comprehensive treatment plan and predict the prognosis of the patient.

\section{CONFLICT OF INTEREST}

No potential conflict of interest relevant to this article was reported.

\section{ORCID}

\author{
Daeun Ko \\ https://orcid.org/0000-0003-1300-8143 \\ Hyun Nam \\ https://orcid.org/0000-0002-5929-4109 \\ Young-Joo Shim \\ https://orcid.org/0000-0001-7514-5974 \\ Jin-Kyu Kang \\ https://orcid.org/0000-0001-7668-7468
}

\section{REFERENCES}

1. Kuribayashi A, Okochi K, Kobayashi K, Kurabayashi T. MRI findings of temporomandibular joints with disk perforation. Oral Surg
Oral Med Oral Pathol Oral Radiol Endod 2008;106:419-425.

2. Shen P, Huo L, Zhang SY, Yang C, Cai XY, Liu XM. Magnetic resonance imaging applied to the diagnosis of perforation of the temporomandibular joint. J Craniomaxillofac Surg 2014;42:874878.

3. Machon V, Levorova J, Hirjak D, Drahos M, Foltan R. Temporomandibular joint disc perforation: a retrospective study. Int J Oral Maxillofac Surg 2017;46:1411-1416.

4. Westesson PL, Larheim TA, Tanaka H. Posterior disc displacement in the temporomandibular joint. J Oral Maxillofac Surg 1998;56:1266-1273; discussion 1273-1274.

5. Nam H, Shim YJ, Kang JK. Acute malocclusion caused by articular disc perforation: a case report. J Oral Med Pain 2018;43:142146.

6. Cholitgul W, Petersson A, Rohlin M, Akerman S. Clinical and radiological findings in temporomandibular joints with disc perforation. Int J Oral Maxillofac Surg 1990;19:220-225.

7. An SY, Jung JK. Fracture of the articular disc in the temporomandibular joint: two case reports. Dentomaxillofac Radiol 2015;44:20140218.

8. Tamimi DF, Hatcher D. Temporomandibular joint. Philadelphia: Elsevier; 2016.

9. Kim JY, Jeon KJ, Kim MG, Park KH, Huh JK. A nomogram for classification of temporomandibular joint disk perforation based on magnetic resonance imaging. Oral Surg Oral Med Oral Pathol Oral Radiol 2018;125:682-692.

10. Massilla Mani F, Sivasubramanian SS. A study of temporomandibular joint osteoarthritis using computed tomographic imaging. Biomed J 2016;39:201-206.

11. Muñoz-Guerra MF, Rodríguez-Campo FJ, Escorial Hernández V, Sánchez-Acedo C, Gil-Díez Usandizaga JL. Temporomandibular joint disc perforation: long-term results after operative arthroscopy. J Oral Maxillofac Surg 2013;71:667-676. 